Revista Colombiana de Obstetricia y Ginecología Vol. 55 No.4 • 2004 • (267-278)

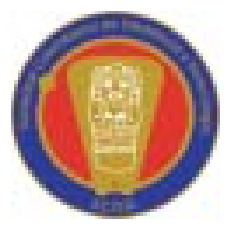

InVESTIGACIÓN ORIGINAL

\title{
ANÁLISIS DE LA MONITORÍA FETAL CON LA TEORÍA DE LA PROBABILIDAD
}

\author{
UISING MATHEMATICAL PROBABILITY THEORY FOR FOETAL \\ MONITORING \\ Javier Rodríguez, M.D.*, Vicente José Carmona, M.D.**, Guillermo Avilán***, Paola \\ Andrea Hincapié**** \\ Recibido: agosto 30/2004 - Revisado: septiembre 21/2004 - Aceptado: octubre 26/2004
}

\section{RESUMEN}

Objetivo: describir la monitoría fetal con la teoría matemática de la probabilidad y la definición de componentes dinámicos del sistema en busca de una primera aproximación en la obtención de mediciones objetivas y reproducibles que permitan evaluar todos los tipos posibles de monitorías obtenidas matemáticamente a partir de una visión general.

Diseño: este es un estudio exploratorio descriptivo con una generalización matemática basada en probabilidades.

Materiales y métodos: se estudiaron 45 mujeres gestantes, quienes se dividieron en dos grupos: el grupo A con factores de riesgo de sufrimiento fetal, y el grupo B sin la presencia de factores de riesgo. A todas se les realizó monitoría fetal y se observó el comportamiento de la frecuencia cardíaca fetal y evaluó en cada registro la aparición y probabilidad de cuatro componentes dinámicos del sistema.

Resultados: son probablemente sanos aquellos fetos cuyas monitorías presentan tres o más veces el componente dinámico del sistema T1, si éste no aparece es tal vez enfermo; en los casos en los que aparece una o dos veces, se define un posible límite entre salud y enfermedad. Las monitorías de fetos posiblemente sanos son las más probables; son 10

* Médico Investigador, Universidad Nacional de Colombia. Bogotá, Colombia. Correo electrónico: grupoinsight2025@yahoo.es

* Ginecólogo Obstetra. Jefe de Medicina Materno Fetal y Unidad de Ultrasonido y Doppler. Hospital Militar Central. Bogotá.

*** Estudiante de Física, Universidad Nacional de Colombia

****Estudiante de Psicología, Universidad Nacional de Colombia. veces más probables que las intermedias y $10^{3}$ veces más probables que las que presentan la menor probabilidad de aparición en el espacio total de probabilidad. Con base en los cálculos matemáticos obtenidos, una de cada dos monitorías del grupo A y una de cada tres del grupo B podrían tener un diagnóstico errado según los parámetros clínicos convencionales. Esta metodología se puede utilizar para evaluar en el tiempo la evolución hacia la salud o la enfermedad en cada paciente. Sin embargo, se requiere un estudio más riguroso para una aplicación clínica.

Palabras clave: bienestar fetal, monitoría fetal, probabilidad.

\section{SUMMARY}

Objective: describing foetal monitoring using mathematical probability theory and defining the system's dynamic components, seeking an initial approach towards obtaining objective and reproducible measurements, such general overview allowing any monitoring trace so obtained to be mathematically evaluated.

Design: this was a descriptive exploratory study employing probability-based mathematical generalisation.

Materials and methods: 45 pregnant women were studied. They were divided into two groups: group A presented foetal suffering risk factors 
whilst group B presented no such risk factors. All of them undergone fetal monitoring; foetal heartbeat behaviour was recorded. Each data-readout was evaluated for the appearance and probability of four dynamic system components (DSC).

Results: a foetus was probably healthy if it had 3 or more times DSC T1 in its readout; if this was not the case, then such foetus was probably sick. Cases where DSC T1 appeared once or twice defined a possible limit between health and illness. It was 10 times more probable that possibly healthy foetuses would be monitored than intermediate traces and 1,000 times more probable than those presenting the least probability of appearing within total probability. The mathematical calculations suggested that one in two readouts from group A and one in three from group B could have had a doubtful diagnosis according to conventional clinical parameters. This methodology could be used for evaluating each patient's evolution towards health or illness. However, a more rigorous study is required for clinical application.

Key words: foetal welfare, foetal monitoring, probability.

\section{INTRODUCCIÓN}

Los métodos de evaluación del bienestar fetal ${ }^{1,2}$ basados en el examen de la frecuencia cardiaca fetal son ampliamente utilizados en la práctica clínica diaria. Estas pruebas estudian la frecuencia cardiaca fetal en reposo o con estímulos específicos como la estimulación vibro acústica.

La monitoría fetal ha tenido diferentes definiciones de los resultados normales ${ }^{3}$ y los criterios para su análisis no son unánimes. ${ }^{4}$ Sin embargo, el esquema de interpretación más utilizado se basa en la aparición de aceleraciones o desaceleraciones con respecto a una línea de base en un periodo dado. Generalmente, una monitoría fetal se considera reactiva si presenta por lo menos dos aceleraciones en 20 minutos y no reactiva si no las desarrolla, si exhibe una taquicardia o bradicardia sostenidas o si aparecen desaceleraciones. A pesar de la existencia de guías para el análisis de este examen, su interpretación es discutida y no reproducible ${ }^{3,4}$.

Para una descripción precisa de la monitoría fetal se necesita la aplicación de una teoría matemática que la evalúe objetivamente. El cambio de la frecuencia cardiaca es un evento que varía continuamente dentro de un rango restringido. Al definir eventos dinámicos discretos en el registro total de la monitoría es posible aplicar la teoría de las probabilidades para obtener una mayor rigurosidad.

La probabilidad es una medida matemática adimensional de la posibilidad de la ocurrencia de un evento. ${ }^{5-7}$ En un experimento que presenta varios resultados posibles o eventos, la frecuencia relativa de un evento particular es el cociente entre la cantidad de veces que se presenta tal resultado y el total de repeticiones del experimento. Un ejemplo típico es el lanzamiento de un dado, que muestra seis resultados posibles. En diez lanzamientos, la frecuencia relativa del resultado «cae la cara 3», es el número de veces que se obtuvo dicha cara dividido entre diez. Se observa que para un número muy grande de lanzamientos, la frecuencia relativa de cada cara del dado tiende a $1 / 6$, que es justamente la medida de la probabilidad. ${ }^{7}$ El espacio de la probabilidad corresponde al conjunto de todos los posibles resultados evaluados con las probabilidades de un experimento en particular. En este trabajo las probabilidades son utilizadas para definir dicho espacio, por lo tanto no se trabaja con probabilidades estadísticas que definan la posibilidad de que una monitoría sea sana o enferma, sino la posibilidad de su existencia, con unos parámetros matemáticos determinados.

El objetivo de la presente investigación es hacer un estudio matemático reproducible de la monitoría fetal, aplicando la teoría de la probabilidad, describiendo objetivamente las monitorías de fetos sanos y enfermos, para construir el espacio total de probabilidades que caracterice cualquier tipo de monitoría. 


\section{MÉTODOS}

Este es un estudio descriptivo exploratorio de la monitoría fetal que incluye una generalización matemática basada en la teoría de la probabilidad. Se estudiaron 45 mujeres gestantes que ingresaron al Servicio de Medicina Materno Fetal del Hospital Militar Central de Bogotá, entre septiembre y diciembre del 2003, con edades gestacionales comprendidas entre 28 y 41 semanas. Se dividieron en dos grupos: grupo A, gestantes que presentaron factores de riesgo, 24 pacientes; grupo B, gestantes que no presentaron factores de riesgo, 21 pacientes. Los detalles de cada grupo se describen en las tablas 1 y 2. Se tomaron monitorías fetales con estrés o sin estrés mínimo de 20 minutos de duración a cada una de las pacientes. La velocidad del papel fue de $3 \mathrm{~cm}$ por minuto y la escala para la frecuencia cardiaca fetal fue de 30 latidos por minuto por centímetro de papel. Para ello se utilizó un monitor electrónico fetal Corometrics. Esta metodología evalúa el bienestar fetal de cada paciente independientemente de la edad gestacional, a partir de 28 semanas, de la posible relación existente entre las enfermedades que presenten la madre o el hijo y de si la prueba es realizada con o sin estrés. Las pruebas fueron valoradas por un ginecoobstetra según los criterios actuales de interpretación, ${ }^{8,9}$ ver definiciones.

Se definieron cuatro componentes dinámicos del sistema (CDS), ver definiciones. Se evaluó cada monitoría midiendo el número de veces que se presentó cada componente dinámico en la totalidad del registro, contando una sola vez los CDS más pequeños dentro de los más grandes y contando una sola vez el T1i, sin importar si la desaceleración es mucho más profunda. En este trabajo no se supone una recta entre dos puntos discontinuos, así que no se pueden evaluar estas regiones de la monitoría. Se calculó la frecuencia de aparición de cada CDS y se analizaron los resultados obtenidos mediante el cálculo de las probabilidades y la comparación de las frecuencias de aparición; finalmente se construyó el espacio total de probabilidad de los componentes dinámicos del sistema a través de la definición de rangos de probabilidad. Una vez clasificados los CDS por rangos de probabilidades y obtenidas las combinaciones posibles, se define el espacio total de probabilidad, que está construido matemáticamente a partir de la totalidad de las posibles monitorías con base en esta forma de evaluación. La probabilidad total de cada tipo de monitoría se calcula multiplicando las probabilidades de los rangos de cada CDS que define cada monitoría. Las comparaciones numéricas en órdenes de magnitud se hacen con el resultado numérico de la probabilidad total. Esta metodología describe de manera objetiva cada componente dinámico del sistema para cualquier monitoría fetal, por lo que no son necesarios análisis estadísticos adicionales.

\section{Definiciones}

Criterios utilizados para la lectura de las monitorías, según la literatura actual. ${ }^{8,9}$

Aceleración: aumento abrupto de la frecuencia cardiaca fetal por lo menos de 15 latidos por minuto, con respecto a la línea de base, durante no menos de 15 segundos. El pico de la aceleración se presenta antes de los primeros 30 segundos del aumento.

Desaceleración: disminución de la frecuencia cardiaca fetal con respecto a la línea de base. Las desaceleraciones tempranas y tardías aparecen de forma gradual, siempre asociadas a una contracción uterina. El nadir de las desaceleraciones tempranas coincide con el pico de la contracción, mientras que el de las tardías se presenta después. Las desaceleraciones variables aparecen de manera abrupta y no se asocian con las contracciones uterinas.

NST reactiva: monitoría sin estrés normal; presencia de por lo menos dos aceleraciones en 20 minutos, sin anomalías en la línea de base ni presencia de desaceleraciones.

NST no reactiva: monitoría sin estrés anormal; ausencia de aceleraciones o aceleraciones inadecuadas por su amplitud, duración o número, taquicardia sostenida, bradicardia sostenida, disminución de la variabilidad o presencia de desaceleraciones periódicas variables o tardías. 


\section{Tabla 1. Pacientes con factores de riesgo (grupo A)}

Paciente Factores de riesgo

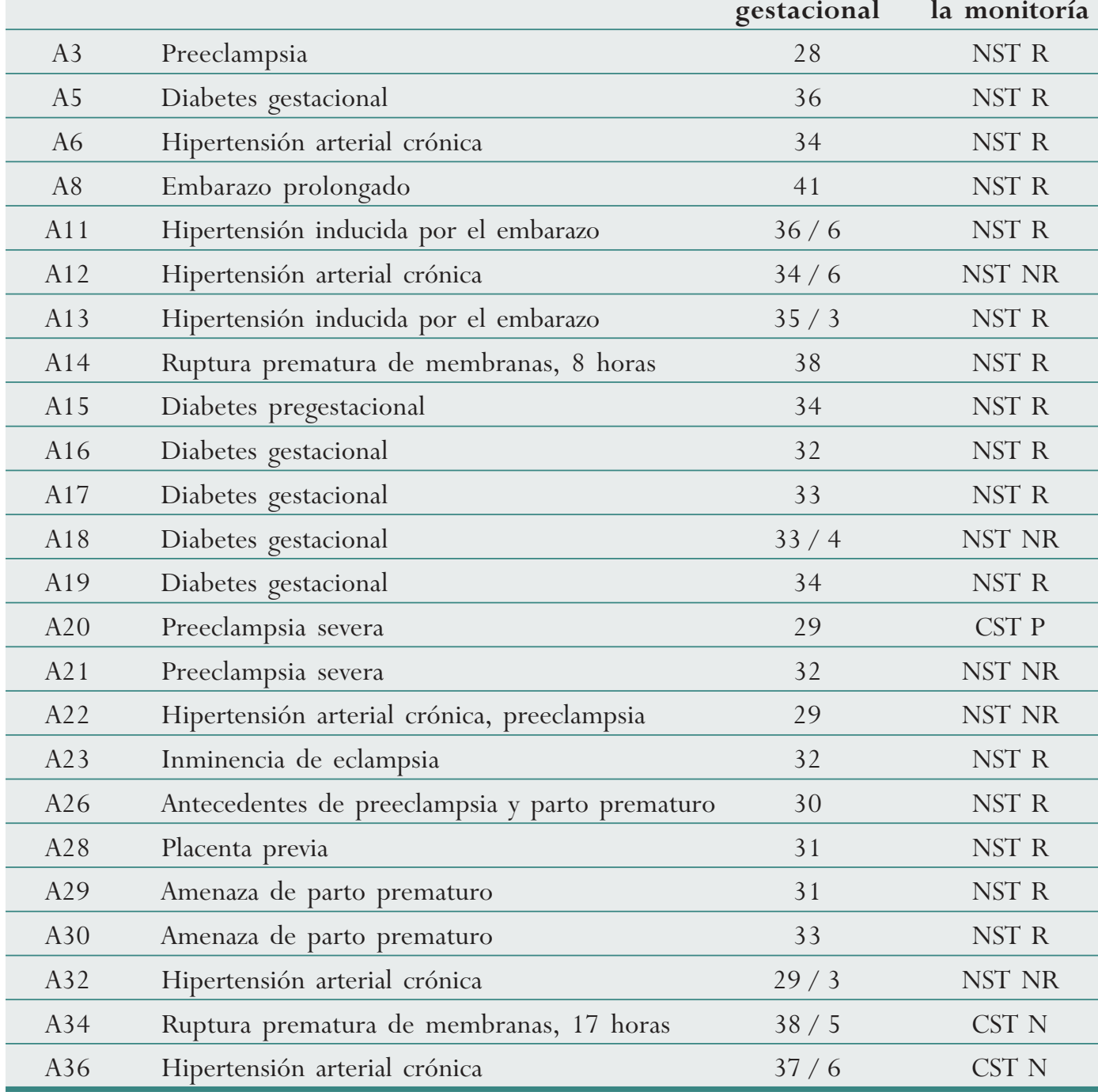

Edad gestacional: tiempo transcurrido en el embarazo en semanas/días. Diagnóstico de la monitoría: NST R: monitoría sin estrés reactiva. NST NR: monitoría sin estrés no reactiva. CST N: monitoría con estrés negativa. CST P: monitoría con estrés positiva.

CST negativa: monitoría con estrés normal; ausencia de desaceleraciones tardías.

CST positiva: monitoría con estrés anormal; más del 50\% de las contracciones se acompañan de desaceleraciones tardías.

\section{Definición de los $\mathrm{CDS}^{\mathrm{a}}$}

Componente dinámico T1, CDS T1: cambio de la frecuencia cardiaca fetal de 20 segundos de base por 15 latidos/minuto de altura.

\footnotetext{
Definición hecha por el primer autor.

El signo menos indica una disminución en la frecuencia cardiaca fetal.
}

\section{Componente dinámico T1 invertido, CDS} T1i: cambio de la frecuencia cardiaca fetal de 20 segundos de base por $(-15)^{\mathrm{b}}$ latidos/minuto de altura.

Componente dinámico T2, CDS T2: cambio de la frecuencia cardiaca fetal de 10 segundos de base por 10 latidos/minuto de altura.

Componente dinámico T3, CDS T3: cambio de la frecuencia cardiaca fetal de 5 segundos de base por 10 latidos/minuto de altura.

La medida de los CDS se realiza respecto al punto anterior del aumento o decrecimiento sin tener en cuenta una línea de base. 
Tabla 2. Pacientes sin factores de riesgo (grupo B)

\begin{tabular}{ccc} 
Paciente & $\begin{array}{c}\text { Edad } \\
\text { gestacional }\end{array}$ & $\begin{array}{c}\text { Diagnóstico } \\
\text { de la monitoría }\end{array}$ \\
\hline B3 & 39 & NST R \\
\hline B4 & $37 / 4$ & CST N \\
\hline B5 & 35 & NST R \\
\hline B7 & 38 & NST R \\
\hline B8 & 39 & NST R \\
\hline B10 & 36 & NST R \\
\hline B13 & 35 & NST R \\
\hline B15 & 40 & NST R \\
\hline B16 & $40 / 6$ & NST NR \\
\hline B20 & 39 & NST R \\
\hline B21 & 33 & NST R \\
\hline B22 & 39 & NST R \\
\hline B23 & 40 & NST R \\
\hline B24 & 40 & NST R \\
\hline B28 & 38 & NST R \\
\hline B29 & 38 & NST NR \\
\hline B30 & $34 / 3$ & NST R \\
\hline B31 & 36 & NST NR \\
\hline B32 & $38 / 6$ & NST R \\
\hline B33 & $38 / 1$ & NST R \\
\hline B34 & $38 / 5$ & NST N \\
\hline
\end{tabular}

Edad gestacional: tiempo transcurrido en el embarazo en semanas/días. Diagnóstico de la monitoría: NST R: monitoría sin estrés reactiva. NST NR: monitoría sin estrés no reactiva. CST N: monitoría con estrés negativa. CST P: monitoría con estrés positiva.

Frecuencia de aparición ${ }^{\mathrm{c}}$ : número de veces que aparece un componente dinámico en la totalidad de la monitoría.

Probabilidad: medida numérica predictiva que toma valores reales entre 0 y 1 , de la posibilidad de que un evento ocurra; la probabilidad 1 indica que el evento ocurrirá con toda seguridad, la probabilidad 0 señala que el evento no ocurrirá.

Probabilidad de un $\mathrm{CDS}^{\mathrm{d}}$ : es el producto del tiempo que tarda cada evento dinámico multiplicado por la frecuencia de aparición del mismo y dividido en el tiempo total de la monitoría.

\footnotetext{
c, d,e Definiciones hechas por el primer autor.

Criterio definido por el primer autor.
}

Probabilidad total $\left(\right.$ PT) ${ }^{\mathrm{e}}$ : la probabilidad total de un tipo de monitoría es el producto de las probabilidades de los rangos de los CDS que la constituyen.

\section{RESULTADOS}

Se evaluó el número de veces que se presentó cada componente dinámico en la totalidad del registro y se calculó la frecuencia de aparición de cada uno. El CDS T1 apareció entre 0 y 8 veces y su probabilidad de aparición osciló entre 0 y 0,1250; el CDS T2 se presentó entre 0 y 20 veces y su probabilidad estuvo entre $0 \mathrm{y}$ 0,1232; el CDS T3 apareció entre 0 y 28 veces y su probabilidad osciló entre 0 y 0,10370; y el CDS T1i se presentó entre 0 y 4 veces y su probabilidad de aparición estuvo entre $0 \mathrm{y}$ 0,0629. Estos resultados se describen en las tablas 3 y 4 .

Son probablemente sanos aquellos fetos cuyas monitorías presentan tres o más veces el CDS T1, obviando las otras condiciones. Los fetos de las monitorías en las que el CDS T1i aparece tres o más veces, a menos que T1 se presente tres o más veces, se consideran posiblemente enfermos. Si el CDS T1 aparece 0 veces el feto podría estar enfermo. En las monitorías en las que el CDS T1 aparece una o dos veces se hace necesario un nuevo parámetro de comparación para determinar su estado: la observación del cociente PT3/PT2 ${ }^{\mathrm{f}}$. Si éste es mayor a 0,7 ; se clasifican como posiblemente sanos, de lo contrario como posiblemente enfermos (tabla 5).

La evaluación matemática, con el cálculo de probabilidades, de las monitorías fetales lleva a la clasificación que se presenta en la tabla $\mathbf{6}$.

A partir de los intervalos de aparición de cada CDS, se definieron rangos de probabilidades. Por ejemplo, la probabilidad total de que el CDS T1 aparezca 0, 1 ó 2 veces es la PT T1 del rango 0-2 (tabla 7). Teniendo las probabilidades por rango de todos los CDS y combinándolas de todas las formas posibles se define el espacio total de proba- 
bilidad que muestra todas las monitorías posibles de acuerdo con esta forma de evaluación (tabla 8).

Las monitorías más probables tienen probabilidad total de 0,02434 y las menos probables 0,0004. En total se obtienen 420 posibles monitorías con esta metodología. Del total se muestran diez: cuatro de las más probables, tres intermedias y las tres menos probables. Para las monitorías más probables PT3/PT2 es mayor que 0,7 ; esto significa que las monitorías más probables son las de fetos posiblemente sanos, éstas son diez veces más probables que las intermedias y $10^{3}$ más probables que las menos frecuentes. Por ejemplo 0,0004 (la probabilidad total de la monitoría menos probable), multiplicada por mil es 0,04 que está en el orden de magnitud de la monitoría más probable. En los grupos A y B, la evaluación matemática fue diferente a la evaluación clínica convencional en trece $(54,16 \%)$ y ocho $(38,1 \%)$ monitorías respectivamente. Al aplicar esta metodología a una misma paciente en el tiempo se podría evaluar la evolución hacia la salud o hacia a la enfermedad de manera más objetiva y reproducible.

\section{DISCUSIÓN}

Este es el primer trabajo donde se aplica una teoría matemática a la monitoría fetal, la teoría de la probabilidad, evaluando los CDS como elementos

\begin{tabular}{cccccccccc}
\multicolumn{7}{c}{ Tabla 3 . Resultados del grupo A } & & & \\
Paciente & Tiempo & F T1 & P T1 & F T2 & P T2 & F T3 & P T3 & F T1i & P T1i \\
\hline A3 & 1.190 & 0 & 0 & 1 & 0,0084 & 1 & 0,0042 & 0 & 0 \\
\hline A5 & 1.480 & 2 & 0,0270 & 3 & 0,0202 & 4 & 0,0135 & 2 & 0,0270 \\
\hline A6 & 1.350 & 1 & 0,0148 & 15 & 0,1111 & 28 & 0,1037 & 3 & 0,0444 \\
\hline A8 & 1.680 & 7 & 0,0833 & 20 & 0,1190 & 27 & 0,0803 & 0 & 0 \\
\hline A11 & 1.330 & 2 & 0,0300 & 2 & 0,0150 & 7 & 0,0263 & 0 & 0 \\
\hline A12 & 1.260 & 0 & 0 & 1 & 0,0079 & 3 & 0,0119 & 0 & 0 \\
\hline A13 & 1.210 & 4 & 0,0661 & 10 & 0,0826 & 15 & 0,0619 & 3 & 0,0495 \\
\hline A14 & 1.530 & 3 & 0,0392 & 10 & 0,0653 & 16 & 0,0522 & 2 & 0,0261 \\
\hline A15 & 1.830 & 2 & 0,0218 & 9 & 0,0491 & 11 & 0,0300 & 1 & 0,0109 \\
\hline A16 & 2.710 & 2 & 0,0147 & 7 & 0,0258 & 14 & 0,0258 & 2 & 0,0147 \\
\hline A17 & 1.200 & 0 & 0 & 4 & 0,0333 & 6 & 0,0250 & 0 & 0 \\
\hline A18 & 1.400 & 0 & 0 & 4 & 0,0285 & 5 & 0,0178 & 0 & 0 \\
\hline A19 & 1.420 & 1 & 0,0140 & 3 & 0,0211 & 7 & 0,0246 & 0 & 0 \\
\hline A20 & 2.490 & 0 & 0 & 1 & 0,0040 & 1 & 0,0020 & 2 & 0,0160 \\
\hline A21 & 1.860 & 0 & 0 & 0 & 0 & 1 & 0,0026 & 0 & 0 \\
\hline A22 & 2.400 & 0 & 0 & 1 & 0,0041 & 2 & 0,0041 & 0 & 0 \\
\hline A23 & 1.950 & 0 & 0 & 5 & 0,0256 & 7 & 0,0179 & 0 & 0 \\
\hline A26 & 1.640 & 8 & 0,0975 & 12 & 0,0731 & 14 & 0,0426 & 1 & 0,0121 \\
\hline A28 & 2.260 & 2 & 0,0176 & 15 & 0,0663 & 27 & 0,0597 & 2 & 0,0176 \\
\hline A29 & 1.290 & 3 & 0,0465 & 9 & 0,0697 & 16 & 0,0620 & 0 & 0 \\
\hline A30 & 1.360 & 2 & 0,0294 & 4 & 0,0294 & 6 & 0,0220 & 0 & 0 \\
\hline A32 & 1.510 & 1 & 0,0132 & 5 & 0,0331 & 5 & 0,0165 & 0 & 0 \\
\hline A34 & 1.300 & 3 & 0,0461 & 8 & 0,0615 & 9 & 0,0346 & 2 & 0,0307 \\
\hline A36 & 1.390 & 0 & 0 & 5 & 0,0359 & 8 & 0,0287 & 0 & 0 \\
\hline
\end{tabular}

Tiempo: duración total en segundos de la monitoría. F indica la frecuencia de aparición del respectivo CDS y $\mathbf{P}$ su probabilidad. 


\begin{tabular}{|c|c|c|c|c|c|c|c|c|c|}
\hline Paciente & Tiempo & F T1 & P T1 & F T2 & P T2 & F T3 & P T3 & F T1i & P T1i \\
\hline B3 & 1.290 & 8 & 0,124 & 15 & 0,1162 & 23 & 0,0891 & 0 & 0 \\
\hline B4 & 1.630 & 1 & 0,0122 & 1 & 0,0061 & 8 & 0,0245 & 0 & 0 \\
\hline B5 & 1.320 & 1 & 0,0151 & 6 & 0,0454 & 15 & 0,0568 & 0 & 0 \\
\hline B7 & 1.440 & 2 & 0,0277 & 4 & 0,0277 & 9 & 0,0312 & 0 & 0 \\
\hline B8 & 1.460 & 5 & 0,0684 & 18 & 0,1232 & 28 & 0,0958 & 2 & 0,0273 \\
\hline $\mathrm{B} 10$ & 1.290 & 5 & 0,0775 & 10 & 0,0775 & 16 & 0,062 & 0 & 0 \\
\hline B13 & 1.860 & 5 & 0,0537 & 10 & 0,0537 & 14 & 0,0376 & 2 & 0,0215 \\
\hline B15 & 1.280 & 8 & 0,125 & 14 & 0,1093 & 15 & 0,0585 & 1 & 0,0156 \\
\hline B16 & 1.250 & 0 & 0 & 0 & 0 & 0 & 0 & 0 & 0 \\
\hline B20 & 1.270 & 4 & 0,0629 & 12 & 0,0944 & 16 & 0,0629 & 4 & 0,0629 \\
\hline B2 1 & 1.240 & 0 & 0 & 4 & 0,0322 & 6 & 0,0241 & 1 & 0,0161 \\
\hline B22 & 1.230 & 5 & 0,0813 & 12 & 0,0975 & 22 & 0,0894 & 0 & 0 \\
\hline $\mathrm{B} 23$ & 1.920 & 4 & 0,0416 & 12 & 0,0625 & 20 & 0,052 & 1 & 0,0104 \\
\hline B24 & 1.370 & 1 & 0,0145 & 6 & 0,0437 & 14 & 0,051 & 0 & 0 \\
\hline B28 & 1.890 & 0 & 0 & 7 & 0,037 & 8 & 0,0211 & 1 & 0,0105 \\
\hline B29 & 1.200 & 0 & 0 & 6 & 0,05 & 14 & 0,0583 & 2 & 0,0333 \\
\hline B30 & 1.520 & 7 & 0,0921 & 16 & 0,1052 & 21 & 0,069 & 2 & 0,0263 \\
\hline B31 & 1.200 & 0 & 0 & 4 & 0,0333 & 5 & 0,0208 & 0 & 0 \\
\hline B32 & 1.290 & 1 & 0,0155 & 2 & 0,0155 & 5 & 0,0193 & 0 & 0 \\
\hline B33 & 2.700 & 8 & 0,0592 & 17 & 0,0629 & 22 & 0,0407 & 1 & 0,0074 \\
\hline B34 & 1.730 & 2 & 0,0231 & 19 & 0,1098 & 26 & 0,0751 & 4 & 0,0462 \\
\hline
\end{tabular}

Tiempo: duración total en segundos de la monitoría. F indica la frecuencia de aparición del respectivo CDS y $\mathbf{P}$ su probabilidad.

y su relación armónica con la totalidad, construyendo una metodología reproducible y objetiva para evaluar cualquier monitoría de gestantes con fetos de edades entre 28 y 41 semanas. Esta nueva forma de medir y evaluar describe el registro, permite una mejor utilización de la información contenida en éste y sugiere un posible límite matemático entre la salud y la enfermedad para las monitorías en las que se presenta una o dos veces el CDS T1. La aparición en el tiempo de CDS en la monitoría de una misma paciente podría evidenciar la evolución de la dinámica cardiaca fetal desde la salud hasta la enfermedad y viceversa. Esta metodología evalúa el bienestar fetal obviando la posible relación existente entre las enfermedades que presenten la madre y/o el hijo; al hacer esto no se está obviando dicha relación, sólo se hace una abstracción simplificadora que permita ver esta interacción como un todo, evitando complicaciones innecesarias en la aplicación de la metodología.

A partir del uso de esta teoría se encuentra que si el número de apariciones del CDS T1 es mayor o igual a tres se presenta como posible marcador de salud y su ausencia total como posible evidencia de enfermedad. Las monitorías con un CDS T1, cuya aparición sea de una o dos veces requieren de un segundo parámetro, que es el cociente PT3/PT2. Si es mayor a 0,7 los fetos se clasifican como posiblemente sanos, de lo contrario como posiblemente enfermos; de este modo PT3/PT2 = 0,7 sugiere un posible límite entre salud y enfermedad. Por ejemplo, las pacientes A19 y A32 tienen una vez el CDS T1 y la relación entre PT3 y PT2 es de 1,16 para la paciente A19 y 0,5 para la paciente A32. Así, el feto de la 


\begin{tabular}{lccc}
\multicolumn{4}{c}{ Tabla 5. Relación PT3/PT2 } \\
para los casos que lo requieren \\
Paciente & PT2 & PT3 & PT3/PT2 \\
\hline A5 & 0,0202 & 0,0135 & 0,6683 \\
\hline A6 & 0,1111 & 0,1037 & 0,9333 \\
\hline A11 & 0,0150 & 0,0263 & 1,7533 \\
\hline A15 & 0,0491 & 0,0300 & 0,6109 \\
\hline A16 & 0,0258 & 0,0258 & 1,0 \\
\hline A19 & 0,0211 & 0,0246 & 1,1658 \\
\hline A28 & 0,0663 & 0,0597 & 0,9004 \\
\hline A30 & 0,0294 & 0,0220 & 0,7482 \\
\hline A32 & 0,0331 & 0,0165 & 0,4984 \\
\hline B4 & 0,0061 & 0,0245 & 4,0163 \\
\hline B5 & 0,0454 & 0,0568 & 1,2511 \\
\hline B7 & 0,0277 & 0,0312 & 1,1263 \\
\hline B24 & 0,0437 & 0,0510 & 1,1670 \\
\hline B32 & 0,0155 & 0,0193 & 1,2451 \\
\hline B34 & 0,1098 & 0,0751 & 0,6839 \\
\hline
\end{tabular}

paciente A19 se clasifica como posiblemente sano y el de la paciente A32 como posiblemente enfermo.

Se obtuvo una cantidad finita de posibles tipos de monitorías, 420 en total, con la metodología teórica desarrollada. Los fetos clasificados como posiblemente sanos por presentar tres o más CDS T1 en el trazado son muy poco probables; sin embargo, las monitorías más probables muestran un cociente PT3/PT2 mayor a 0,7; por lo tanto, las monitorías más probables son de fetos posiblemente sanos. Las monitorías más probables son diez veces más probables que las intermedias y mil veces más probables que las menos probables.

Para realizar este trabajo se tuvo en cuenta la mirada teórica, matemática y física de los sistemas dinámicos y los fractales sobre salud y enfermedad. Con este tipo de descripción teórica se evidencia que el concepto homeostático de línea de base no es apropiado, puesto que las medidas realizadas de la frecuencia cardiaca fetal muestran que ésta varía de manera impredecible. En los cálculos realizados se observa que las monitorías más probables son las que satisfacen el criterio para ser posiblemente sano, lo cual corresponde con lo observado globalmente, pues en general hay más fetos sanos que enfermos. Esto ratifica el estudio realizado, ya que los resultados de cualquier estudio teórico que busque describir la realidad deben corresponder con las observaciones experimentales.

El procedimiento habitual para la interpretación de las monitorías se basa en la observación de características aisladas —aceleraciones y desaceleraciones - con respecto a la línea de base y no en un análisis de la totalidad del registro. La forma actual de evaluación hace uso de criterios que son poco precisos. En esta investigación se estudia la variabilidad de la frecuencia cardiaca fetal con medidas objetivas en un marco matemático general para mayor rigurosidad del estudio.

Al examinar cuidadosamente el trazado de las monitorías, se observó que además de los cambios de la frecuencia cardiaca fetal definidos en las guías de interpretación actuales como aceleraciones y desaceleraciones, existen otros tipos de cambios que se repiten a lo largo del trazado, los cuales permiten una evaluación y aproximación más global de la monitoría fetal en el tiempo. De todas estas variaciones se escogieron cuatro tipos básicos de cambios de la frecuencia cardiaca fetal por su simplicidad. Cada una de estas variaciones se definió teniendo en cuenta su amplitud tomada en latidos por minuto y su duración en segundos; así se definieron los CDS. Estos se construyeron para el estudio de la variabilidad de la frecuencia cardiaca fetal; no tienen correspondencia alguna con los conceptos de aceleración o desaceleración y son independientes de una línea de base, de la edad gestacional, siempre y cuando ésta sea mayor a 28 semanas y del procedimiento elegido, con estrés o sin éste.

Con base en los diagnósticos clínicos relacionados con las monitorías y observando las frecuencias de aparición de los componentes dinámicos, se evidenció que en la preeclampsia el componente dinámico CDS T1 no apareció y que en monitorías consideradas sanas con la metodología convencional, asociadas con una respuesta clínica favorable, este componente se presentó de cuatro a ocho veces. Sin embargo, la importancia de este hallazgo debe aclararse en investigaciones posteriores. 
Tabla 6. Evaluación de las monitorías con las probabilidades

\section{Posiblemente sanos}

1 ó 2 T1 Posiblemente sanos según PT3/PT2

Posible enfermedad según PT3/PT2

Posiblemente enfermos
A8, A13, A14, A26, A29, A34, B3, B8,B10, B13, B15, B20, B22, B23, B30, B33.

A6, A11, A16, A19, A28, A30, B4, B5,B7, B24, B32.

A5, A15, A32, B34.

A3, A12, A17, A18, A20, A21, A22,A23, A36, B16, B21, B28, B29, B31.

Tabla 7. Probabilidad de cada CDS por rangos

\begin{tabular}{lccccccc}
$\begin{array}{l}\text { Rangos } \\
\text { de T1 }\end{array}$ & PT T1 & $\begin{array}{c}\text { Rangos } \\
\text { de T2 }\end{array}$ & PT T2 & $\begin{array}{c}\text { Rangos } \\
\text { de T3 }\end{array}$ & PT T3 & $\begin{array}{c}\text { Rangos } \\
\text { de T1i }\end{array}$ & PT T1i \\
\hline $0-2$ & 0,644444 & $0-2$ & 0,2000 & $0-2$ & 0,1111 & $0-1$ & 0,7727 \\
\hline $3-5$ & 0,222222 & $3-5$ & 0,2444 & $3-5$ & 0,1333 & $2-4$ & 0,2272 \\
\hline $6-8$ & 0,133333 & $6-8$ & 0,1333 & $6-8$ & 0,2000 & & \\
\hline & $9-11$ & 0,1333 & $9-11$ & 0,0667 & & \\
\hline & $12-14$ & 0,1111 & $12-14$ & 0,1111 & & \\
\hline & $15-17$ & 0,1111 & $15-17$ & 0,1556 & & \\
\hline & $18-20$ & 0,0667 & $18-20$ & 0,0222 & & \\
\hline & & & $21-23$ & 0,0889 & \\
\hline
\end{tabular}

PT: probabilidad total para cada CDS en cada rango.

Tabla 8. Síntesis del espacio total de probabilidad

\begin{tabular}{lcccccccc}
$\begin{array}{l}\text { Rango } \\
\text { T1 }\end{array}$ & PT1 & $\begin{array}{c}\text { Rango } \\
\text { T2 }\end{array}$ & PT2 & $\begin{array}{c}\text { Rango } \\
\text { T3 }\end{array}$ & PT3 & $\begin{array}{c}\text { Rango } \\
\text { T1inv }\end{array}$ & $\begin{array}{c}\text { PT1i } \\
\text { Probabilidad } \\
\text { total }\end{array}$ \\
\hline $0-2$ & 0,6444 & $3-5$ & 0,2444 & $6-8$ & 0,2000 & $0-1$ & 0,7727 & 0,02434 \\
\hline $0-2$ & 0,6444 & $0-2$ & 0,2000 & $15-17$ & 0,1556 & $0-1$ & 0,7727 & 0,01549 \\
\hline $0-2$ & 0,6444 & $9-11$ & 0,1333 & $6-8$ & 0,2000 & $0-1$ & 0,7727 & 0,01327 \\
\hline $0-2$ & 0,6444 & $3-5$ & 0,2444 & $21-23$ & 0,0889 & $0-1$ & 0,7727 & 0,01082 \\
\hline $0-2$ & 0,6444 & $6-8$ & 0,1333 & $24-26$ & 0,0222 & $0-1$ & 0,7727 & 0,00147 \\
\hline $3-5$ & 0,2222 & $3-5$ & 0,2444 & $12-14$ & 0,1111 & $2-4$ & 0,2272 & 0,00137 \\
\hline $3-5$ & 0,2222 & $15-20$ & 0,1111 & $9-11$ & 0,0667 & $0-1$ & 0,7727 & 0,00127 \\
\hline $6-8$ & 0,1333 & $6-8$ & 0,1333 & $24-26$ & 0,0222 & $2-4$ & 0,2272 & 0,00008 \\
\hline $6-8$ & 0,1333 & $15-23$ & 0,1111 & $18-20$ & 0,0222 & $2-4$ & 0,2272 & 0,00007 \\
\hline $6-8$ & 0,1333 & $18-20$ & 0,0667 & $24-26$ & 0,0222 & $2-4$ & 0,2272 & 0,00004 \\
\hline
\end{tabular}

En la tabla se muestran las probabilidades para diez tipos de monitorías de las 420 posibles. Las primeras cuatro filas son cuatro de los tipos de monitorías más probables, las tres siguientes son de los intermedios y las tres últimas son $10^{3}$ menos probables que las primeras. 
Las interrupciones en el trazado de las monitorías no permiten la medición del registro en su totalidad; si se presentan discontinuidades de más de 5 segundos de duración podría haber algunos CDS no medidos. Si se omite este hecho en el análisis se pueden cometer errores en la medición, para hacerla correctamente se necesita una calidad en el registro que permita una evaluación continua de por lo menos 20 minutos; de esta forma se asegura la precisión y coherencia de los resultados. Se recomienda la obtención de la monitoría sin discontinuidades para la aplicación de esta metodología.

Al tener las frecuencias de aparición de los componentes dinámicos de las 45 monitorías estudiadas, se observa que existen unos intervalos restringidos para su aparición. Por ejemplo, el CDS T1 aparece de 0 a 8 veces y difícilmente se saldrá de este intervalo. Así, es posible calcular las probabilidades de todas las posibles frecuencias de aparición de cada CDS y desarrollar el espacio total de probabilidad en el que se muestran todas las monitorías posibles con base en este análisis. En la presente investigación se ha hecho una simplificación en la que se ha descrito la monitoría fetal únicamente con cuatro componentes dinámicos del sistema.

Recientemente se aplicaron teorías de la matemática y de la física, como la teoría del caos y la geometría fractal, al estudio de la salud y la enfermedad con resultados concluyentes. Basados en estas teorías Goldberger y cols. ${ }^{10-14}$ analizaron la dinámica cardiaca y encontraron caracterizaciones matemáticas para la salud y la enfermedad. En estos estudios, se asocia la enfermedad con la periodicidad y con la excesiva variabilidad del sistema, mientras que la salud se asocia con una variabilidad intermedia entre los dos anteriores posibles estados del sistema. Huikuri y cols. ${ }^{15}$ calcularon medidas fractales objetivas de la dinámica cardiaca en pacientes con infarto agudo de miocardio, con una fracción de eyección menor al 35\%, superando los factores predictores convencionales de muerte. Con metodologías propias basadas en teorías matemáticas, Rodríguez y cols. ${ }^{16}$ lograron una diferenciación matemática estricta entre la salud y la enfermedad en un modelo experimental de reestenosis coronaria en porcinos, sin la necesidad de validaciones estadísticas como en esta investigación.

Este es un estudio descriptivo exploratorio de la monitoría fetal basado en una teoría matemática; los componentes dinámicos del sistema se evalúan objetivamente para cualquier monitoría fetal. Al comparar la aparición de los CDS en una monitoría y observar las relaciones que se presentan entre las diferentes monitorías y respecto a la totalidad, se deducen reglas generales de posible diferenciación de salud y enfermedad que muestran unas relaciones matemáticas para cada monitoría particular y para la totalidad. Esta conclusión se logra al buscar generalizaciones de observaciones particulares de los CDS, sin necesidad de análisis estadísticos.

Por ser este un trabajo teórico-práctico se partió de concepciones previas basadas en las teorías del caos aplicadas a la dinámica cardiaca y a la fisiología en general. Goldberger ${ }^{14}$, en contra del punto de vista homeostático, planteó que un registro muy plano o muy aleatorio podría ser asociado con la enfermedad y que los registros que están entre estos dos comportamientos podrían relacionarse con la salud; de esta forma se escogieron cuatro monitorías: un registro con muy poca variabilidad, casi plano, otro con aceleraciones y desaceleraciones aleatorias pronunciadas y dos monitorías que presentan un comportamiento intermedio entre estos dos extremos. Las dos primeras confirmadas como enfermas y las últimas consideradas sanas por el diagnóstico clínico convencional y por los resultados prácticos en la clínica, con las cuales se realiza la generalización matemática.

Con las cuatro monitorías seleccionadas ya se pueden encontrar los criterios que diferencian salud de enfermedad y anticipar el resultado del estudio; con el aumento del número de pacientes se confirma dicho resultado, de tal forma que incrementar el número de pacientes estudiadas sólo confirma lo calculado previamente. Mediante la evaluación de la totalidad de los pacientes incluidos en el estudio se construye el espacio total de pro- 
babilidades, el cual incluye todos los posibles tipos de monitorías que se pueden definir con la metodología diseñada, se encuentra un conjunto finito de posibles monitorías. Tanto los parámetros de evaluación como el espacio total de probabilidades son resultados del estudio que confirmaron lo previsto por la concepción teórica.

Mediante la aplicación de esta nueva metodología se encontraron diferencias con los parámetros de evaluación convencionales. En los grupos A y $\mathrm{B}$, la interpretación de los registros fue diferente en trece $(54,16 \%)$ y ocho $(38,1 \%)$ monitorías respectivamente. En el grupo con factores de riesgo, cuatro monitorías interpretadas clínicamente como sanas - A3, A17, A23 y A36- se incluyeron en el grupo de las posiblemente enfermas por no presentar CDS T1 y dos monitorías reactivas -A5 y A15- se incluyeron en el grupo de posible enfermedad por presentar una relación PT3/ PT2 menor que 0,7. Igualmente, en el grupo B, las monitorías de las pacientes B21 y B28 leídas como reactivas ingresaron al grupo de posiblemente enfermas. Once monitorías normales de ambos grupos -A6, A11, A16, A19, A28, A30 y B4, B5, B7, B24 y B32 - cumplieron con el criterio de PT3/PT2 y se clasifican como posiblemente sanas y dos monitorías no reactivas -A32 y B34 - por medio de este mismo criterio se clasificaron como posiblemente enfermas. El resultado matemático muestra que la evaluación clínica convencional podría fallar en una de cada dos monitorías del grupo con factores de riesgo y en una de cada tres sin factores de riesgo.

Este trabajo es una observación global de la monitoría fetal. Para hacer observaciones más precisas hace falta definir todos los posibles tipos de componentes dinámicos del sistema, calcular sus probabilidades y aclarar su impacto clínico. Respecto a todos los posibles componentes dinámicos del sistema probablemente sería muy importante la construcción de sistemas electrónicos, que permitan una evaluación más precisa y objetiva de las medidas de cambio de la frecuencia cardiaca fetal, lo cual daría información más completa para una aplicación eficaz de esta metodología, así como para la implementación de otras teorías como la del caos.

Este trabajo propone una mirada, una metodología y una aplicación teórica originales independientes de las vías de aproximación convencionales o de investigación actuales. En este estudio no se consideró una relación entre los criterios desarrollados y las observaciones clínicas finales de evaluación neonatal, limitación que será tenida en cuenta en posteriores investigaciones con esta misma metodología. Además de crear una nueva medida para un examen comúnmente utilizado, se propone una forma original de evaluación que debe ser mejorada para tener una aplicación clínica.

\section{CONCLUSIONES}

1. Los fetos cuyas monitorías presentan tres o más veces el CDS T1, obviando las otras condiciones son probablemente sanos. Si el CDS T1 aparece 0 veces, el feto está probablemente enfermo. Los fetos de las monitorías en las que el CDS T1i aparece tres o más veces, a menos que el CDS T1 se presente tres o más veces, se consideran probablemente enfermos. Monitorías con un CDS T1 de uno o dos y con una proporción PT3/PT2 mayor de 0,7 son posiblemente sanos. Si esta proporción es menor de 0,7 son posiblemente enfermos.

2. En la evaluación diagnóstica de cada monitoría se encuentran diferencias para cada grupo de estudio comparada con la evaluación clínica convencional. El diagnóstico fue diferente en trece monitorías $(54,16 \%)$ del grupo A y ocho $(38,1 \%)$ del grupo B. La evaluación clínica convencional podría fallar en una de cada dos monitorías del grupo con factores de riesgo y en una de cada tres sin factores de riesgo.

3. Esta metodología se puede aplicar tomando monitorías en el tiempo para una misma paciente, evidenciando probablemente el desarrollo de la dinámica cardiaca fetal desde la salud hasta la enfermedad y viceversa. 
4. En órdenes de magnitud son diez veces más probables las monitorías de fetos posiblemente sanos comparadas con monitorías de aparición intermedias y $10^{3}$ veces más probables que las más infrecuentes.

5. La cantidad de monitorías fetales son finitas dentro del espacio total de probabilidad, en el que se encuentran las 420 posibilidades de todas las monitorías sanas y enfermas con los componentes dinámicos evaluados. Este nuevo espacio permite una mejor utilización de la información contenida en el registro en su medición y sugiere un posible límite matemático entre salud y enfermedad, en órdenes de magnitud son diez veces más probables las monitorías de fetos posiblemente sanos comparadas con monitorías de aparición intermedias y $10^{3}$ veces más probables que las más infrecuentes.

6. Para el refinamiento en la aplicación clínica de la metodología propuesta sería conveniente contrastarla con el resultado perinatal.

\section{AGRADECIMIENTOS}

A todos nuestros profesores desde la primaria hasta la Universidad. A Ary Goldberger y Heikki Huikuri por sus trabajos pioneros de teorías físicas y matemáticas aplicadas a la medicina, que se convirtieron en la única estrella en una noche completamente oscura para nuestro grupo de investigación. Al doctor Jorge Lemus, cardiólogo y profesor de medicina del Hospital Militar Central, por el apoyo al desarrollo de esta investigación.

\section{REFERENCIAS}

1. Benson R. Diagnóstico y tratamiento ginecoobstétrico. 4 ed. México DF: El Manual Moderno SA de CV; 1986.

2. Sánchez F. Alto riesgo obstétrico. Bogotá: Universidad Nacional de Colombia; 1988.

3. Pritchard JA, MacDonald PC, Gant NF (eds). Williams Obstetricia. Barcelona: Salvat Editores; 1986.
4. Borgatta L, Shrout PE, Divon MY. Reliability and reproducibility of nonstress test readings. Am J Obstet Gynecol 1988;159:554-8.

5. Feynman RP, Leighton RB, Sands M. Probabilidad. En: Feynman RP, Leighton RB, Sands M. Física. Vol. 1. Wilmington: Addison-Wesley Iberoamericana, S.A.; 1964. p. 6-1, 6-16.

6. Mood AM, Graybill FA, Boes D. Introduction to the theory of statistics. 3a. ed. Singapore: McGraw-Hill; 1974.

7. Blanco L. Probabilidad, notas de clase. Universidad Nacional de Colombia. Departamento de Matemáticas y Estadística; 1996.

8. Devoe LD. Nonstress testing and contraction stress testing. Obstet Gynecol Clin North Am 1999;26:535-56.

9. National Institute of Child Health and Human Development Research Planning Workshop. Electronic fetal heart rate monitoring: research guidelines for interpretation. Am J Obstet Gynecol 1997;177:1385-90.

10. Goldberger AL, West BJ. Fractals in physiology and medicine. Yale J Biol Med 1987;60:421-35.

11. Goldberger AL, Rigney DR, West BJ. Chaos and fractals in human physiology. Sci Am 1990;262:42-9.

12. Lipsitz LA, Goldberger AL. Loss of «complexity» and aging. Potential applications of fractals and chaos theory to senescence. JAMA 1992;267:1806-9.

13. Goldberger AL. Non-linear dynamics for clinicians: chaos theory, fractals, and complexity at the bedside. Lancet 1996;347:1312-4.

14. Goldberger AL, Amaral LA, Hausdorff JM, Ivanov PCh, Peng CK, Stanley HE. Fractal dynamics in physiology: alterations with disease and aging. Proc Natl Acad Sci USA 2002;99:2466-72.

15.Huikuri HV, Mäkikallio TH, Peng CK, Goldberger AL, Hintze U, Moller M. Fractal correlation properties of R-R interval dynamics and mortality in patients with depressed left ventricular function after an acute myocardial infarction. Circulation 2000;101:47-53.

16. Rodríguez J, Mariño M, Avilán N, Echeverri D. Medidas fractales de arterias coronarias en un modelo experimental de reestenosis. Armonía matemática intrínseca de la estructura arterial. Rev Colomb Cardiol 2002;10:65-72. 